\title{
Eleven-Year Experience with the Avidin-Biotin Pretargeting System in Glioblastoma: Toxicity, Efficacy and Survival
}

\author{
Chiara M. Grana ${ }^{1}$, Marco Chinol ${ }^{1}$, Concetta De Cicco ${ }^{1}$, Mirco Bartolomei ${ }^{3}$, Marta Cremonesi ${ }^{2}$, \\ Lisa Bodei ${ }^{1}$, Paola A. Rocca ${ }^{1}$, Monica Pacifici ${ }^{1}$, Simone Tiberini ${ }^{1}$, Silvia M. Baio ${ }^{1}$, \\ Giovanni Broggi ${ }^{4}$, Stefano Severi ${ }^{5}$ and Giovanni Paganelli ${ }^{*}, 1$
}

\author{
${ }^{1}$ Divisions of ${ }^{1}$ Nuclear Medicine and, ${ }^{2}$ Medical Physics, European Institute of Oncology, via Ripamonti 435, 20141 \\ Milan, Italy \\ ${ }^{3}$ Division of Nuclear Medicine, M. Bufalini Hospital, viale Ghirotti 286, 47023 Cesena (FC), Italy \\ ${ }^{4}$ Department of Neurosurgery, Fondazione IRCCS Istituto Neurologico C. Besta, via Celoria 11, 20133 Milan, Italy \\ ${ }^{5}$ Radiometabolic Therapy Unit, Istituto Scientifico Romagnolo per lo Studio e la Cura dei Tumori, via Maroncelli 34, \\ 47014 Meldola (FC), Italy
}

\begin{abstract}
Background: The 3-step avidin-biotin pretargeting approach is applied in patients with recurrent glioblastoma (GBM), using biotinylated anti-tenascin monoclonal antibody as the first step of pretargeting followed by avidin and ${ }^{90} \mathrm{Y}$ biotin.

Methods: The present study reviews objective response and overall survival rates in 502 glioblastoma patients treated with 3-step radioimmunotherapy in our institute from December 1994 to December 2005. Patients underwent standard treatment before receiving Pretargeted Antibody-Guided Radionuclide Therapy with ${ }^{90}$ Y-biotin (PAGRIT ${ }^{\mathbb{B}}$ ).

Results: Of the 502 patients, 272 (54\%) were evaluable for response and 375 (75\%) for overall survival. 174 patients $(64 \%)$ continued to progress after PAGRIT ${ }^{\circledR}, 77(28 \%)$ obtained disease stabilization, and $21(8 \%)$ showed objective tumor regression. Survival of the 375 evaluable patients was $98.4 \%$ at 6 months, $79.2 \%$ at 12 months, $51.7 \%$ at 18 months, and $30.7 \%$ at 24 months after the first cycle of PAGRIT ${ }^{\mathbb{B}}$. All 375 received 3-step PAGRIT ${ }^{\mathbb{B}}$ at recurrence of GBM. The median survival time from diagnosis was 19 months.

Conclusion: The results from this retrospective analysis suggest that ${ }^{90} \mathrm{Y}$-biotin PAGRIT ${ }^{\circledR}$ interferes with the progression of glioblastoma, prolonging survival in a larger number of patients. Our analysis forms the basis for further prospective trials, where radioimmunotherapy, which is known to be more effective in minimal residual disease, could be offered immediately after surgery.
\end{abstract}

Keywords: Avidin-biotin, brain tumor, glioblastoma, monoclonal antibodies, pretargeting, radioimmunotherapy, tenascin.

\section{INTRODUCTION}

Our experience at the European Institute of Oncology (Milan, Italy) of radioimmunotherapy (RIT) and the avidinbiotin system for the treatment of high-grade gliomas (anaplastic astrocytoma and glioblastoma) began at the end of 1994, with the administration of RIT in a patient with recurrent anaplastic astrocytoma, who obtained a complete remission [1]. This patient is currently alive, 16 years after treatment. Thus, after this encouraging, albeit anecdotal result, the development of a 3-step RIT approach followed the conventional pathway of any new radiopharmaceutical.

In 1999 we published the $1^{\text {st }}$ Phase I-II study, evaluating the toxicity of the avidin-biotin pretargeting approach in 48 patients with histologically confirmed high-grade glioma

*Address correspondence to this author at the Division of Nuclear Medicine, European Institute of Oncology, Via Ripamonti 435, 20141 Milano, Italy; Tel: +39 0257489 043; Fax: +39 02 94379223;

E-mail: divisione.medicinanucleare@ieo.it
(HGG) [2]. In this trial we showed that 3-step RIT with ${ }^{90} \mathrm{Y}$ biotin produced limited toxicity up to the activity of 60 $\mathrm{mCi} / \mathrm{m}^{2}$ due to the favorable biodistribution of ${ }^{90} \mathrm{Y}$-DOTAbiotin. Objective therapeutic responses evaluated according to WHO criteria were also documented in $25 \%$ of these patients.

The results obtained in the phase I-II study prompted us to apply the same approach in an adjuvant setting. Thirtyseven patients with high-grade glioma (glioblastoma and anaplastic astrocytoma) and no evidence of disease after surgery and radiotherapy underwent, one month after completing the conventional treatment, treatment with Y-90biotin according to the previous phase I-II protocol. The median disease-free interval was 28 months and median survival 33.5 months for patients with glioblastoma. In the grade III glioma group, the median disease-free interval was 56 months (range: 15-60) [3]. Unfortunately, this study was interrupted because budget restrictions prevented further purchase of clinical grade monoclonal antibodies avidin and ${ }^{90} \mathrm{Y}$-biotin, needed for the study. 
In another phase I-II study we investigated the safety profile and antitumor efficacy of locoregional RIT in recurrent high-grade glioma after second surgical debulking with implantation of an indwelling catheter in the surgical cavity. The maximum tolerated activity was $1110 \mathrm{GBq}$ limited by neurologic toxicity. Again, an overall objective response was obtained in $25 \%$ of patients, with stable disease in 50\% [4]. Following these results, we carried out a trial of locoregional radioimmunotherapy associated with oral chemotherapy (temozolomide) in order to achieve synergistic activity by combining radioactivity with chemotherapy. $75 \%$ of GBM patients obtained disease stabilisation, with a overall survival of 25 months and a progression free survival (PFS) of 10 months [5].

More recently, in cooperation with the Neuro-Oncology Department of the National Neurological Institute "C. Besta" in Milan [6], we evaluated a combined therapeutic strategy: twenty-six patients with recurrent GBM were treated with local delivery of chemotherapy (mitoxantrone) and local pretargeted RIT through an indwelling catheter in the surgical cavity. PFS after second surgery for the entire group was, at 6 and 12 months, $61 \%$ and $22 \%$, respectively, while survival after recurrence was $80 \%, 53 \%$ and $42 \%$ at 6,12 and 18 months, respectively. The percentage of long-term survivors was very high: $42 \%$ of patients were still alive 18 months after the second surgery.

What these trials have in common is the fact that all the patients received RIT on the basis of a pretargeting strategy based on anti-tenascin monoclonal antibody and the avidinbiotin system with ${ }^{90} \mathrm{Y}$ as the radioactive source $[1,7]$. In addition to these trials (all sponsored by Academic grants only), we treated numerous other recurrent glioblastoma patients, in progression after standard treatments, on a compassionate basis.

The aim of the present study was to retrospectively evaluate the efficacy of 3-step-PAGRIT ${ }^{\circledR}$ in terms of overall survival in a large population of patients with recurrent glioblastoma.

\section{MATERIALS AND METHODS}

The studies involved were approved by the Ethical Committee of the European Institute of Oncology and all patients, after being informed of the nature, aim and potential risks of the therapy, signed a consent form before starting therapy. Inclusion criteria were the same as described in our previous work [2]; in particular: the presence of recurrent/relapsed, tenascin-positive glioblastoma; baseline morphological evaluation with CT (Computed Tomography) scan or MRI (Magnetic Resonance Imaging); preserved bone marrow and kidney function; Karnofsky performance status (KPS) $>60 \%$. Exclusion criteria were: KPS $<60$; the presence of a known second cancer; history of allergic or anaphylactic reactions and pregnancy.

\section{Patients}

Detailed patient characteristics are reported in Table $\mathbf{1 .}$

As there is no Neurosurgery Unit at our Institute, the majority of patients were referred to us by colleagues from other cancer centers. A certain number patients, all in progression after standard treatments, arrived of their own volition after finding out about our institute via the Internet. Patients received RIT at disease progression, documented by a CT scan or MRI performed one month before therapy. Patients received RIT about 3-6 months after diagnosis: before RIT they were treated with surgery, conventional radiotherapy, and in few cases with chemotherapy.

Table 1. Detailed Patients' Characteristics

\begin{tabular}{|l|r|}
\hline Treated patients & 502 \\
\hline Males & 316 \\
\hline Females & 186 \\
\hline Age range (yrs) & $18-82$ \\
\hline $\mathrm{N}^{\circ}$ pts receiving CT & 289 \\
\hline KPS & $>60$ \\
\hline Pts evaluable for response & $272 / 502$ \\
\hline Pts evaluable for survival & $375 / 502$ \\
\hline
\end{tabular}

Routine blood and liver and renal function tests were performed before and after RIT. Antibodies against murine antibodies, avidin and streptavidin were assayed in serum 30-40 days after RIT. Check-ups, including clinical examination (evaluation of performance status, quality of life, number of seizures, drug intake) and CT or MRI scans were scheduled at three-month intervals starting, when possible, two months after the end of RIT.

From December 1994 to December 2005 we treated 502 glioblastoma patients with 3-step pre-targeted antibody guided radioimmunotherapy. All patients had previously received surgery (complete or only large biopsy) and radiotherapy; 289 had also received chemotherapy (platinum-based chemotherapy or, more recently, temozolomide) for histologically confirmed glioblastoma in progression at the time of RIT. 186 were females and 316 were males (age range 18 - 82 years).

See Fig. (1) for the 3-step pretargeting approach.

\section{Radiolabelling and Administration Protocols}

The radiolabelling procedure and pretargeting protocol were performed as previously described [2, 3].

The biotinylated MoAbs were injected first, followed 2436 hours later by the administration of avidin and streptavidin. Patients remained under medical observation for an hour following administration to monitor possible reactions.

Ten minutes before the injection of radioactive biotin, 20 $\mathrm{mg}$ of biotinylated human serum albumin was administered i.v. to reduce circulating levels of streptavidin (second chase).

To allow scintigraphic monitoring of radiolabel localisation, the therapeutic activity was mixed with 74-111 $\mathrm{MBq}$ of the gamma source indium- 111 bound to $0.05 \mathrm{mg}$ of DOTA-biotin. The intravenous injected activity ranged from 2.22 to $2.97 \mathrm{GBq} / \mathrm{m} 2(60-80 \mathrm{mCi} / \mathrm{m} 2)$ and was administered 24 hours after avidin and streptavidin infusion. 

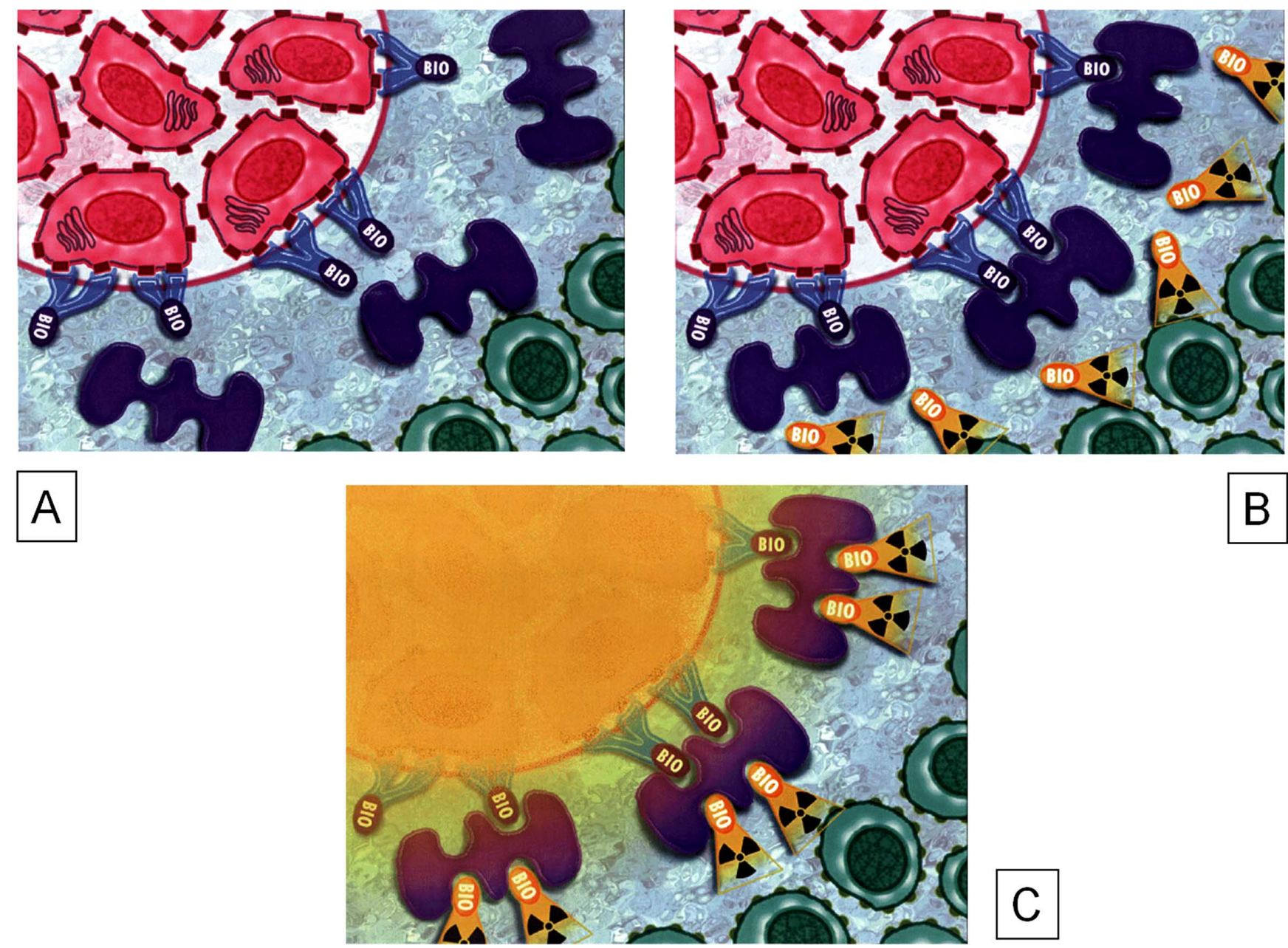

Fig. (1). The pre-targeting approach (3-step).

\section{Data Analysis and Statistical Evaluation}

The objective therapeutic response was evaluated with CT or MRI images of the brain before and after treatment. The first post-treatment evaluation was performed 60 days later and repeated every 2-3 months thereafter, when possible.

On the basis of these criteria and the WHO score, patients were classified as follows: $100 \%$ reduction: complete response $(\mathrm{CR}),>50 \%$ reduction: partial response $(\mathrm{PR}), \quad<25 \%$ reduction: stable disease (SD), tumor enlargement $>25 \%$ : progressive disease (PD).

From March to July 2008, 502 enquiries were sent to the relevant Town Registries of Births, Deaths and Marriages asking for an update on patient status. A Kaplan-Meier estimation of survival and log-rank tests were applied.

Statistical analyses were performed with SPSS 10.0 for Windows computer software.

\section{RESULTS}

\section{Toxicity and Quality of Life}

Treatment was well-tolerated in all patients, without serious acute or late side-effects. A small number of patients who had previously received monoclonal antibodies and avidin for diagnostic purposes developed a mild allergic reaction (in particular, skin rash) during administration. No side-effects occurred from the biotinylated $\mathrm{MoAb}$ or ${ }^{90} \mathrm{Y}$ biotin injections.

As previously described [2, 3], no renal or liver alterations were observed during follow-up. As regards hematological toxicity, the nadir of haematological values occurred 3 to 4 weeks after treatment and began with thrombocytopenia. Full recovery was observed in all patients after 4-6 weeks. No cases of fever, infection or bleeding, or other significant sequelae were reported.

\section{Immunological Response}

The presence of antibodies against the injected reagents was assayed before and after treatment. Almost all patients (90\%) developed anti-streptavidin antibodies, $70 \%$ developed anti-avidin antibodies, and 20\% developed antibodies against the murine MoAbs. These results are very similar to data published previously by our group; in particular, they confirm the low frequency of reaction to murine MoAbs and the high immunogenicity of streptavidin [8].

\section{Therapeutic Effect}

More than $75 \%$ of patients reduced or suspended steroids in the 2-4 months following treatment. 
Therapeutic effects and data on survival are shown in Figs. (2, 3).

272 of the 502 treated patients were evaluable for therapeutic response on the basis of WHO criteria. We could assess only 272 out of 502 patients as the majority lived far away from our hospital and were lost to follow up.

When evaluating therapeutic response to RIT, we had to take into consideration a number of factors regarding our

\begin{tabular}{|lll|}
\hline \multicolumn{2}{|c|}{ Glioblastoma PTS } & \\
& $\mathrm{N}^{\circ}$ & $\%$ \\
PR & 21 & 8 \\
SD & 77 & 28 \\
PD & 174 & 64 \\
\hline
\end{tabular}

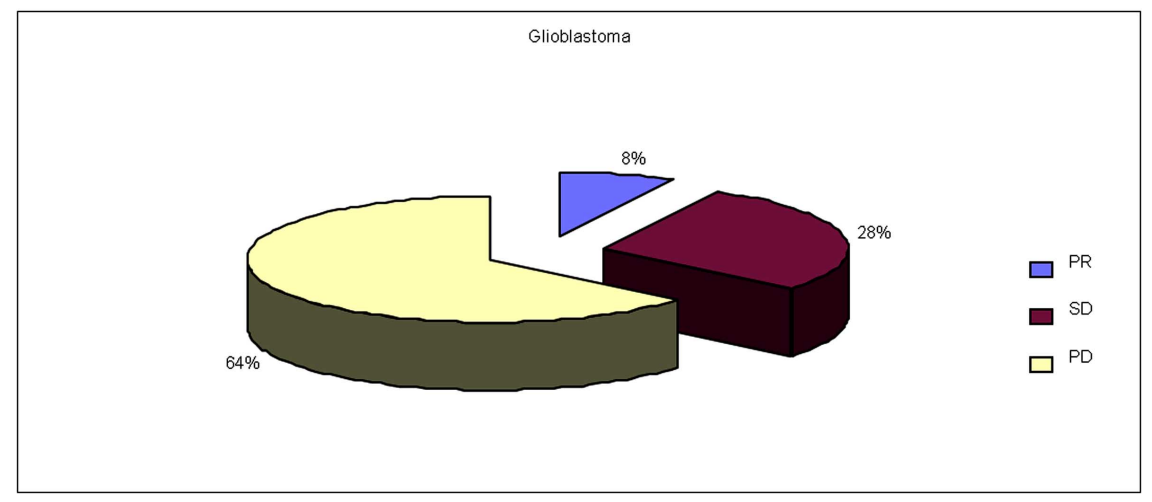

Fig. (2). Objective responses.

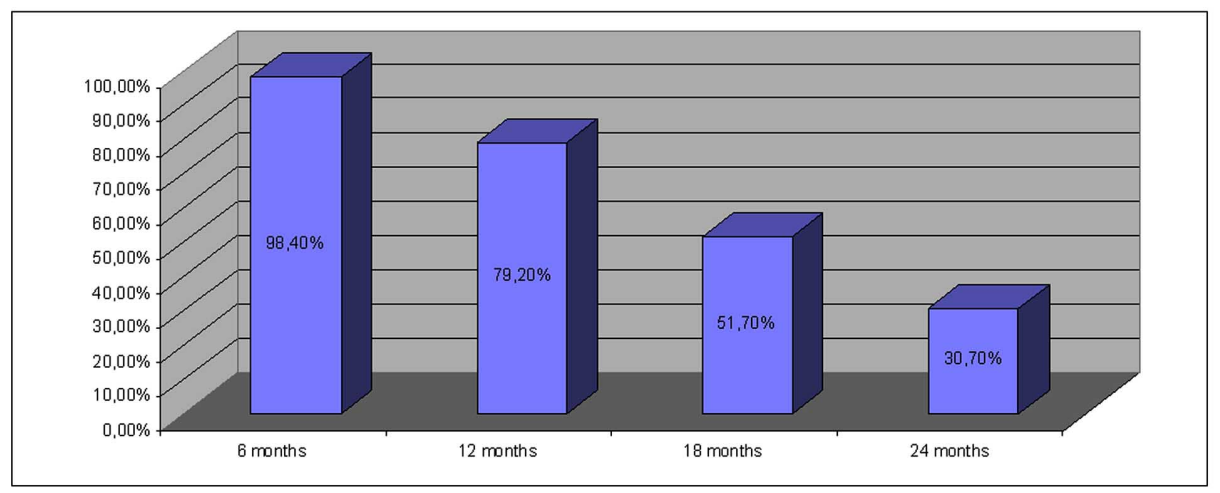

Median survival time from diagnosis: 19 months

\section{Cumulative Survival}

\begin{tabular}{|ll|}
\hline Cumulative survival & \\
Time & $\%$ \\
6 months & 98.4 \\
12 months & 79.2 \\
18 months & 51.7 \\
24 months & 30.7 \\
\hline
\end{tabular}

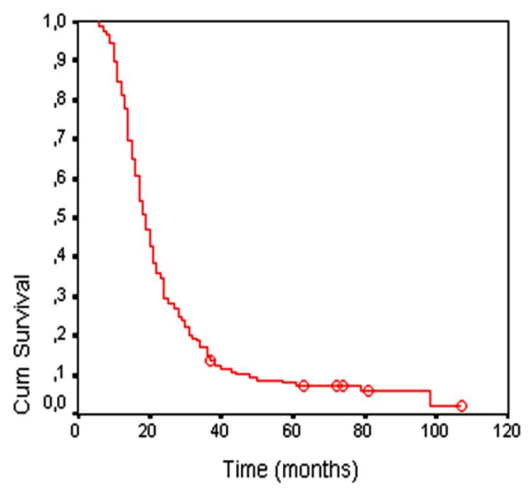

Fig. (3). Survival data. 
patients: heterogeneous population; different disease extension; different KPS at enrolment; and different tumor tenascin expression. Despite such diversity, interesting results were obtained: 21 patients $(8 \%)$ showed a partial response (PR) at CT or MRI scans. 77 patients $(28 \%)$ obtained disease stabilization and the remaining 174 patients $(64 \%)$ had progressive disease at follow up.

In the PR group, 15 patients were in rapid progression after previous treatments (surgery and radiotherapy), while the remaining 6 patients showed slower disease progression. Duration of response was 13 months in the former PR group and 23 months in the latter. Median survival from diagnosis in this PR group was 26 months, with no side-effects from RIT and with good quality of life.

\section{Survival}

502 enquiries were sent to the relevant Town Registries of Births, Deaths and Marriages asking for an update on patient status, with a response obtained for 375 (75\%); in July 2008, the median survival time from diagnosis was 19 months (95\% CI 18 - 29). GBM cumulative survival was $98.4 \% \pm 0.9 \%$ at 6 months, $79.2 \% \pm 2.1 \%$ at 12 months, $51.7 \% \pm 2.6 \%$ at 18 months, and $30.7 \% \pm 2.4 \%$ at 24 months (Fig. 3).

\section{DISCUSSION}

Glioblastoma remains a major challenge in oncology. A population-based study of newly-diagnosed GBM patients reported an overall survival of only $42.4 \%$ at 6 months, $17.7 \%$ at 1 year and $3.3 \%$ at 2 years, despite surgery, radiotherapy and chemotherapy [9]. However, a more recent, randomized, multinational Phase III study showed that temozolomide improves outcome when administered in an integrative schedule during and following radiotherapy with respect to radiotherapy alone [10]. Salvage therapies after conventional treatments have proven to be ineffective, hence there is no accepted standard second-line care for recurrent glioblastoma patients.

Our 3-step pretargeting PAGRIT ${ }^{\circledR}$ approach was offered to 502 glioblastoma patients from December 1994 to December 2005. All 502 patients, in disease progression after conventional treatments such as surgery, radiotherapy and chemotherapy, were referred to us from other hospitals; PAGRIT $^{\circledR}$ was proposed on a compassionate basis. Extension of surgery, kind of radiotherapy and/or chemotherapy received, and tumor molecular characteristics were not used as eligibility criteria (as in pilot phase I-II trials). We offered 3-step PAGRIT $^{\circledR}$ to all glioblastoma patients with radiological evidence of disease progression, tenascin expression and KPS $>60$.

Some interesting points emerge from our retrospective evaluation of this large population.

First, all patients tolerated the treatment very well, without acute or late side-effects. The low toxicity of high activities of ${ }^{90} \mathrm{Y}$-biotin using a 3 -step pretargeting strategy shows an important advantage over the use of directly labeled antibodies [11] in that the radiolabelled material is cleared quickly from the blood-pool, has favorable biodistribution and is rapidly excreted via the kidneys. In this group of patients we confirmed, on a small sample of patients, dosimetric data that we reported in the study of 1999 [2]: absorbed doses were 2.7 $\pm 1.6 \mathrm{cGy} / 37 \mathrm{MBq}$ in kidneys, $1.5 \pm 1.0 \mathrm{cGy} / 37 \mathrm{MBq}$ in liver and $0.6 \pm 0.3 \mathrm{cGy} / 37$ $\mathrm{MBq}$ in brain, while the dose delivered to the tumour was $15.20 \pm 8.70 \mathrm{cGy} / 37 \mathrm{MBq}$.

Second, although we recruited a heterogeneous population for the 3-step RIT, which could be considered a limit of our study, we are nevertheless the only Nuclear Medicine Department to have administered this therapy with the pretargeting system in such a large group (502) of patients with recurrent glioblastoma.

Third, a therapeutic response was obtained in $21(8 \%)$ patients and disease stabilization in 77 (28\%). All 98 patients obtained an interruption of disease progression, which is significant where recurrent glioblastoma is concerned. However, these results are different from those previously described, where objective responses (complete and partial response) were observed in $29 \%$ of patients, with a further $48 \%$ obtaining disease stabilization. We believe that this could be due to the different populations evaluated, especially if the KPS of enrolled patients, which was globally lower in the present retrospective study, is taken into consideration. This would be interesting to study in depth and could lead to the administration of RIT in an earlier phase of glioblastoma treatment, with patients in better clinical conditions.

Fourth, our most important finding concerned survival. Median overall survival from diagnosis was 19 months (in contrast to the 16 months observed in 31 patients reported in our first paper), with $30.7 \%$ of the patients still alive 2 years after RIT (Fig. 3).

Similar results were described by Stupp and coworkers in 2009 [10] who published the long-term findings of the EORT-NCI phase III trial in glioblastoma patients randomised to receive external radiotherapy alone versus external radiotherapy plus temozolomide. Overall survival was $27.2 \%(95 \%$ CI 22.2-32.5) at 2 years. However, in Stupp's study, treatment was administered at the beginning of the disease, whereas our patient population was in progression after surgery, radiotherapy and chemotherapy (the majority having received different, less active antiblastic drugs, e.g. platinum-based chemotherapy).

Other important results in terms of objective responses and overall survival in the treatment of glioblastoma, were obtained from other studies concerning bevacizumab, the best characterized antiangiogenic therapy, recently approved by FDA as a single agent for the treatment of patients with recurrent GBM following prior upfront, TMZ-based chemoradiotherapy $[12,13]$. Overall, treatment with bevacizumab in multiple GBM studies appears to be well tolerated with toxicity similar to that seen with other solid cancers treated with bevacizumab-containing therapies. Because of the extensive clinical experience with bevacizumab, practical issues regarding its administration, safety profile, and response to treatment have been described [14-16]. However, several important questions about the use of bevacizumab in GBM still remain unanswered, for example, the optimal therapeutic dosage, treatment schedule, treatment duration in responding patients and radiographic response criteria of bevacizumab are all unknown. 
Another important issue, that we have to consider, is that there is no consensus as to why the use of bevacizumab yields resistance to future treatments. Some investigators have found that patients with progression after bevacizumab developed perivascular fibrosis, which limited the amount of subsequent drug delivery to the tumor [17].

However, as bevacizumab continues to be studied, it is evident that although there is an increase in PFS there is no change in overall survival ( 9 months in different studies) as compared with historical controls [18]; significative differences are also evident if we look at the results of our population, that can be considered similar for enrolment criteria.

The interesting results from our retrospective study, albeit not statistically comparable with those of other clinical trials, would seem to indicate that PAGRIT ${ }^{\circledR}$ should be administered earlier in the glioblastoma treatment flowchart. In fact, when we administered RIT in an adjuvant setting after surgery, radiotherapy and chemotherapy [3], we obtained a median disease-free interval of 28 months with a median survival of 33.5 months.

3-step radioimmunotherapy is a multistep process designed to improve the efficiency of radiolabelled MoAb localization to tumor cells [19]. Typically, the MoAb is administered first and an appropriate time interval is allowed to permit the MoAb to localize and bind to its target, after which a radiolabelled, low-molecular-weight vehicle, is administered. The most frequently applied approach capitalizes on the extraordinarily high binding affinity of avidin for biotin. With this three-step process, glioma patients first receive biotinylated anti-tenascin MoAbs followed 24 hours later by avidin and then, approximately 18 hours later, by the administration of an ${ }^{90}$ Y-labeled biotin conjugate. In our study, this approach was well tolerated by all patients, without any discomfort or acute or subacute side-effects.

Tenascin-C, a large, extracellular matrix glycoprotein, is widely expressed in the stroma and mesenchyme of tissues at various stages of differentiation. Increased tenascin- $\mathrm{C}$ expression occurs in a variety of pathologic states, including inflammation and wound healing, and in some tumors, such as breast, squamous cell, lung and prostate carcinomas, melanoma and malignant glioma [20-22]. Tenascin- $\mathrm{C}$ is expressed in more than $90 \%$ of gliomas and its expression increases with increasing tumor grade. Although its functions remain controversial, recent studies suggest that it plays a role in several cellular processes, including adhesion, migration and proliferation [23-28].

In our study of a heterogeneous population of glioblastoma patients, we confirmed that 3-step radioimmunotherapy using the biotinylated anti-tenascin $\mathrm{MoAb}$ and the avidin-biotin system, is a safe therapy offering a clinical benefit in terms of improved survival. No enrolled patient experienced dose-limiting toxicity after the administration of ${ }^{90} \mathrm{Y}$ - biotin.

These results could form the basis for further prospective trials, including those exploiting new radioisotopes, such as Lu-177 or alpha emitters, and provide a means to assess timing and scheduling of radioimmunotherapy in the therapeutic algorithm of high-grade glioma patients. They also open up numerous interesting possibilities for prospective evaluations, especially with regard to new treatment strategies.

Recently, our group developed a new tissue-pretargeted radionuclide treatment method called intraoperative avidination for radionuclide treatment $\left(\right.$ IART $\left.^{\circledR}\right)$. This approach is based on the intraoperative injection of avidin in surgical margins and tumor-surrounding tissues, followed, within 24 hours, by intravenous injection of radioactive biotin-DOTA. Clinical studies have shown that "avidination"' is a simple, well-tolerated and effective way of directing the localization of radioactive biotin. Tissue avidination, as in IART $^{\circledR}$, allows the creation of a transient artificial receptor for biotin and relies on the previously described tumor tropism of avidin [29-31]. This approach, already tested in breast cancer by our group, has been shown to be a safe procedure, delivering about $20 \mathrm{~Gy}$ to the tumor bed after a single i.v. injection of $3.7 \mathrm{GBq}$ of ${ }^{90} \mathrm{Y}$-biotin [3234].

The same principle can be applied in glioblastoma patients; neurosurgeons, immediately after removal (complete or partial) of the tumour, inject avidin into the tumour bed, and the next day the patient receives radiolabelled biotin i.v. as an anticipated boost to EBRT.

Patients normally receive external beam radiotherapy one month after surgery, thus giving glioblastoma cells sufficient time to spread into surrounding tissues. The use of IART $^{\circledR}$ in brain tumours can provide significant accelerating brain irradiation before starting the traditional treatment, thus offering a potential advantage in terms of time to progression and survival. It may, in fact, be advantageous to administer the radioisotopic treatment immediately after debulking surgery in order to exploit the greater permeability of the blood-brain barrier at that time. This would be expected to expose more malignant cells to the radionuclide and hence limit local and distant spread of the cancer.

\section{CONCLUSION}

Glioblastoma multiforme, the most common primary tumor of the central nervous system, continues to have an unacceptable outcome.

Salvage therapies for malignant glioma patients with recurrent disease remain ineffective; such patients therefore represent a major challenge in neuroncology.

Radioimmunotherapy involving the administration of monoclonal antibodies and radioisotopes, in particular using the avidin-biotin system $\left(\right.$ PAGRIT $\left.^{\circledR}\right)$, represents an innovative therapeutic strategy to improve local tumor control and overall outcome when administered as part of a multimodality therapeutic regimen including traditional external-beam radiotherapy, systemic chemotherapy, and innovative treatments; this combined approach can be considered the most effective treatment currently available. Our hope is that the combination of surgery, radiotherapy, chemotherapy, new drugs and PAGRIT $^{\circledR}$ may provide a way of increasing life expectancy in glioblastoma patients.

\section{ACKNOWLEDGEMENTS}

This study was supported by grants from the Italian Association for Cancer Research (AIRC). 
The authors thank William Russel Edu and Grainne Tierney for their help with the English and Deborah Console for editing the manuscript.

\section{CONFLICTS OF INTEREST STATEMENT}

The authors declare that they have no conflict of interest.

\section{REFERENCES}

[1] Goldenberg DM, Sharkey RM, Paganelli G, Barbet J, Chatal JF. Antibody pretargeting advances cancer radioimmunodetection and radioimmunotherapy. J Clin Oncol 2006; 24(5): 82-34.

[2] Paganelli G, Grana C, Chinol M, et al. Antibody-guided three-step therapy for high grade glioma with yttrium-90 biotin. Eur J Nucl Med 1999; 26(4): 348-57.

[3] Grana C, Chinol M, Robertson $\mathrm{C}$, et al. Pretargeted adjuvant radioimmunotherapy with yttrium-90-biotin in malignant glioma patients: a pilot study. Br J Cancer 2002; 86: 207-12.

[4] Paganelli G, Bartolomei M, Ferrari M, et al. Pre-targeted locoregional radioimmunotherapy with $90 \mathrm{Y}$-biotin in glioma patients: phase I study and preliminary therapeutic results. Cancer Biother Radiopharm 2001; 16: 227-35.

[5] Bartolomei M, Mazzetta C, Handkiewicz-Junak D, et al. Combined treatment of glioblastoma patients with locoregional pre-targeted 90 Y-biotin radioimmunotherapy and temozolomide. Q J Nucl Med Mol Imaging 2004; 48(3): 220-8.

[6] Boiardi A, Bartolomei M, Silvani A, et al. Intratumoral delivery of mitoxantrone in association with $90-\mathrm{Y}$ radioimmunotherapy (RIT) in recurrent glioblastoma. J Neurooncol 2005; 72(2): 125-31.

[7] Boerman OC, van Schaijk FG, Oyen WJ, Corstens FH. Pretargeted Radioimmunotherapy of Cancer: Progress Step by Step. J Nucl Med 2003; 44: 400-11.

[8] Paganelli G, Chinol M, Maggiolo M, et al. The three-step pretargeting approach reduces the human anti-mouse antibody response in patients submitted to radioimmunoscintigraphy and radioimmunotherapy. Eur J Nucl Med 1997; 24(3): 350-1.

[9] Ohgaki H, Dessen P, Jourde B, et al. Genetic pathways to glioblastoma: a population-based study. Cancer Res 2004; 64(19): 6892-9.

[10] Stupp R, Hegi ME, Mason WP, et al.; European Organisation for Research and Treatment of Cancer Brain Tumour and Radiation Oncology Groups. National Cancer Institute of Canada Clinical Trials Group. Effects of radiotherapy with concomitant and adjuvant temozolomide versus radiotherapy alone on survival in glioblastoma in a randomised phase III study: 5-year analysis of the EORTC-NCIC trial. Lancet Oncol 2009; 10(5): 459-66.

[11] Paganelli G, Riva P, Deleide G, et al. In vivo labelling of biotinylated monoclonal antibodies by radioactive avidin: a strategy to increase tumour radiolocalisation. Int J Cancer 1988; 2: $121-5$.

[12] National Comprehensive Cancer Network. NCCN practice guidelines in oncology: Central nervous system cancers. v.1.2008. Fort Washington, PA: National Comprehensive Cancer Network, 2009. Available from URL:http://www.nccn.org/professionals/phys ician_gls/PDF/cns.pdf [accessed May 21, 2009].

[13] Avastin [package insert]. South San Francisco, CA: Genentech, Inc, May 2009.

[14] Van Meir EG, Hadjpanayis CG, Norden AD, Shu HK, Wen PY, Olson JJ. Exciting new advances in neuro-oncology: the avenue to a cure for a malignant glioma. CA Cancer J Clin 2010; 60(3): 16693.

[15] Norden AD, Drappatz J, Wen PY. Antiangiogenic therapies for high-grade glioma. Nat Rev Neurol 2009; 5(11): 610-20.
[16] Prados M, Cloughesy T, Samant M, et al. Response as a predictor of survival in patients with recurrent glioblastoma treated with bevacizumab. NeuroOncol 2011; 13(1): 143-51.

[17] de Groot J, Lamborn K, Chang S, et al. Phase II study of aflibercept in recurrent malignant glioma: a North American Brain Tumor Consortium study. J Clin Oncol 2011; 29(19): 2689-95.

[18] Norden A, Drappatz J, Muzikansky A, et al. An exploratory survival analysis of anti-angiogenic therapy for recurrent malignant glioma. J Neurooncol 2009; 92(2): 149-55.

[19] Reilly RM. Radioimmunotherapy of solid tumors: the promise of pretargeting strategies using bispecific antibodies and radiolabeled haptens. J Nucl Med 2006; 47(2): 196-9.

[20] Bourdon MA, Wikstrand CJ, Furthmayr H, Matthews TJ, Bigner DD. Human glioma-mesenchymal extracellular matrix antigen defined by monoclonal antibody. Cancer Res 1983; 43(6): 2796805.

[21] Bourdon MA, Matthews TJ, Pizzo SV, Bigner DD. Immunochemical and biochemical characterization of a gliomaassociated extracellular matrix glycoprotein. J Cell Biochem 1985; 28: 183-95.

[22] Howeedy AA, Virtanen I, Laitinen L, Gould NS, Koukoulis GK, Gould VE. Differential distribution of tenascin in the normal, hyperplastic, and neoplastic breast. Lab Invest 1990; 63(6): 798806.

[23] Pas J, Wyszko E, Rolle K, et al. Analysis of structure and function of tenascin-C. Int J Biochem Cell Biol 2006; 38(9): 1594-602.

[24] Borsi L, Carnemolla B, Nicolo G, Spina B, Tanara G, Zardi L. Expression of different tenascin isoforms in normal, hyperplastic and neoplastic human breast tissues. Int J Cancer 1992; 52(5): 68892.

[25] Ventimiglia JB, Wikstrand CJ, Ostrowski LE, Bourdon MA, Lightner VA, Bigner DD. Tenascin expression in human glioma cell lines and normal tissues. J Neuroimmunol 1992; 36(1): 41-55.

[26] Zagzag D, Friedlander DR, Miller DC, et al. Tenascin expression in astrocytomas correlates with angiogenesis. Cancer Res 1995; 55(4): 907-14.

[27] Herold-Mende C, Mueller MM, Bonsanto MM, Schmitt HP, Kunze $\mathrm{S}$, Steiner HH. Clinical impact and functional aspects of tenascin-C expression during glioma progression. Int J Cancer 2002; 98(3): 362-9.

[28] Behrem S, Zarkovic K, Eskinja N, Jonjic N. Distribution pattern of tenascin-C in glioblastoma: correlation with angiogenesis and tumor cell proliferation. Pathol Oncol Res 2005; 11(4): 229-35.

[29] Yao Z, Zhang M, Sakahara H, Saga T, Arano Y, Konishi J. Avidin targeting of intraperitoneal tumor xenografts. J Natl Cancer Inst 1998; 90(1): 25-9.

[30] Yao Z, Zhang M, Sakahara $\mathrm{H}$, et al. The relationship of glycosylation and isoelectric point with tumor accumulation of avidin. J Nucl Med 1999; 40(3): 479-83.

[31] Chinol M, De Cobelli O, Trifiro ' G, et al. Localization of avidin in superficial bladder cancer: A potentially new approach for radionuclide therapy. Eur Urol 2003; 44: 556-9.

[32] Paganelli G, Ferrari M, Cremonesi M, et al. IART ${ }^{\circledR}$ : Intraoperative avidination for radionuclide treatment. A new way of partial breast irradiation. Breast 2007; 16: 17-26.

[33] Paganelli G, Ferrari M, Ravasi L, et al. Intraoperative avidination for radionuclide therapy: A prospective new development to accelerate radiotherapy in breast cancer. Clin Cancer Res 2007; 13(Suppl 18): 5646s-51s.

[34] Paganelli G, De Cicco C, Ferrari ME, et al. Intraoperative avidination for radionuclide treatment as a radiotherapy boost in breast cancer: results of a phase II study with (90)Y-labeled biotin. Eur J Nucl Med Mol Imaging 2010; 36: 203-11. 